In Situ

Revue des patrimoines

\section{In Situ}

Revue des patrimoines

18 | 2012

Le cheval et ses patrimoines (1ère partie)

\title{
Le cheval, un animal contraint
}

\section{Christophe Degueurce}

\section{OpenEdition}

Journals

Édition électronique

URL : http://journals.openedition.org/insitu/9674

DOI : 10.4000/insitu.9674

ISSN : 1630-7305

\section{Éditeur}

Ministère de la culture

\section{Référence électronique}

Christophe Degueurce, « Le cheval, un animal contraint », In Situ [En ligne], 18 | 2012, mis en ligne le 01 août 2012, consulté le 19 avril 2019. URL : http://journals.openedition.org/insitu/9674 ; DOI : 10.4000/ insitu. 9674

Ce document a été généré automatiquement le 19 avril 2019

\section{(c) $(1) \odot$}

In Situ Revues des patrimoines est mis à disposition selon les termes de la licence Creative Commons Attribution - Pas d'Utilisation Commerciale - Pas de Modification 4.0 International. 


\title{
Le cheval, un animal contraint
}

\author{
Christophe Degueurce
}

1 L'équitation lie deux êtres bien différents dans un mouvement commun, dans une quête $\mathrm{d}^{\prime}$ harmonie qui les transforme en une forme de centaure ${ }^{1}$. Cette fusion tend à gommer les différences existant entre l'homme et l'animal, et la tentation est forte pour le cavalier de voir dans sa monture un autre lui-même, soumis finalement aux mêmes impératifs. Et pourtant, l'espèce équine s'est développée dans un contexte zoologique très spécifique, marqué par une spécialisation dans les activités locomotrices, ces mêmes aptitudes que nous recherchons tant, cette efficacité étant contrebalancée par des contraintes remarquables ${ }^{2}$.

2 L'objectif de cette communication est d'envisager cet animal sous l'angle de ses adaptations et des limites qui en sont le corollaire, ceci afin de porter un regard renouvelé sur une espèce dont nous usons à des fins d'équitation sans souvent bien la connaitre. Cet herbivore occupe une place de proie dans le règne animal. Sa survie dans un milieu ouvert, sauvage, implique la détection des prédateurs et le recours à une stratégie de fuite et/ou de défense. En simplifiant à l'extrême le propos, on peut dire qu'il est doté de capacités sensorielles fines et très différentes des nôtres, et qu'il a développé une remarquable capacité à la course. Nous essaierons de percevoir quel usage nous faisons de ces aptitudes et verrons si les limites qu'elles imposent bornent simplement ces usages ou si, au contraire, elles nous ouvrent des champs de confrontation dans la pratique équestre.

\section{Des perceptions sensorielles différentes des nôtres}

La vision du cheval est assez différente de la nôtre. Nos yeux placés à l'avant de notre face nous apportent une vision stéréoscopique très détaillée mais a pour corollaire la réduction extrême de notre champ de vision. Chez le cheval, les yeux sont placés sur les côtés de la tête et lui apportent deux types de visions, mono- ou binoculaire. Il possède tout d'abord un champ de vision monoculaire très étendu, représentant $142,5^{\circ}$ de chaque côté, à tel point que son champ aveugle est réduit à la ligne du dos, représentant un angle de $5^{\circ}$, et à une zone placée sous sa tête. Ceci lui permet de voir ce qui se passe autour de 
lui, de détecter les mouvements de prédateurs ou de congénères; ces champs monoculaires ne permettent pas une vision de qualité et notamment une bonne perception des reliefs, indispensable à la mise en place d'une réaction. Son champ binoculaire complète donc l'ensemble; il est réduit, représente un angle de $70^{\circ}$ et se trouverait en avant de son chanfrein. Structurellement, la très faible importance de cette vision binoculaire est attestée par la décussation ${ }^{3}$ restreinte des fibres du nerf optique, de l'ordre de 12 à $15 \%$. Un autre élément remarquable est la présence, au niveau de la rétine, d'une bande horizontale, étroite, où la densité des récepteurs est plus importante ; c'est sur cette bande, analogue à la fovea ${ }^{5}$ de l'homme, que la vision est la plus nette, les parties de l'image formées en dehors de cette bande étant floues ${ }^{6}$. Les grands champs monoculaires lui permettent d'avoir une vision panoramique, avec une image limitée en hauteur, et donc de percevoir simultanément deux spectacles différents sans appréciation sûre des distances. Son acuité visuelle est aussi 40 \% inférieure à la nôtre?

L'olfaction est par ailleurs sans conteste plus développée que chez nous et son audition assez proche de la nôtre, à ceci près que la mobilité de ses oreilles lui confère une remarquable capacité de localisation de l'origine des sons.

\section{Un squelette spécialisé pour la course}

$5 \quad$ Le squelette des équidés est marqué par des adaptations à la course. Sa tête est appendue à une encolure longue et souple. Sa colonne thoracique est formée de dix-huit vertèbres là où la majeure partie des espèces possède treize vertèbres; ces vertèbres sont courtes, maintenues par de puissants ligaments qui assurent une forte cohésion de l'ensemble et lient tous les segments entre eux. La colonne lombaire est formée de six vertèbres dont les processus articulaires sont très engainants et dont les deux dernières sont liées entre elles et au sacrum par des articulations intertransversaires. L'ensemble forme un pont thoraco-lombaire très rigide qui permet le soutien du cavalier et de la masse viscérale très volumineuse, et sert de transmission à l'avant-main des forces propulsives exprimées par les membres pelviens. L'encolure est soutenue par un fort ligament nuchal se prolongeant par un ligament supra-épineux solidarisant les sommets des processus épineux, et ainsi l'ensemble de la colonne vertébrale.

6 Les membres sont longs et marqués par une simplification du nombre de rayons osseux. Le membre thoracique ne présente pas de clavicule et est lié au thorax par une puissante sangle musculaire liant la scapula et l'humérus au sternum, aux côtes et à la colonne, ce qui limite fortement les mouvements d'abduction du membre (écartement du plan médian). Surtout, la plupart des articulations des membres thoraciques et pelviens ont des surfaces articulaires condylaires, c'est-à-dire ayant une forme de cylindres favorisant de façon quasi-exclusive les mouvements de flexion-extension dans le plan sagittal (fig. $\mathbf{n}$ $\left.{ }^{\circ} \mathbf{1}\right)$. Chaque articulation condylaire favorise ainsi la flexion et l'extension au détriment des mouvements de rotation externe/interne et des mouvements d'abduction/adduction. Dans le même esprit, les avant-bras et la main sont bloqués en pronation par la fusion de l'ulna et du radius. Les mouvements sont donc essentiellement développés dans le plan sagittal, et les mouvements latéraux très réduits. 
Figure 1

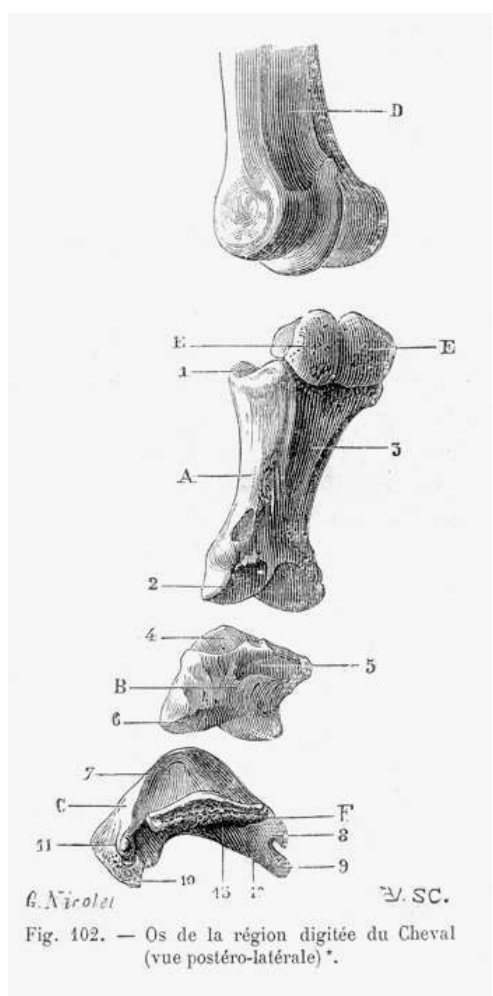

Les phalanges du cheval sont marquées par des surfaces articulaires condylaires qui limitent fortement les mouvements autres que la flexion et l'extension, selon CHAUVEAU, A., ARLOING, S., LESBRE, F.-X. Traité d'anatomie comparée des animaux domestiques. Tome 1. Paris : Baillières, 1905, figure 102.

7 Le membre pelvien est lié au bassin par l'articulation de la hanche, articulation qui a généralement beaucoup de degrés de liberté. Là encore, les mouvements autres que la flexion et l'extension sont limités par la présence d'un ligament supplémentaire, le ligament accessoire de la tête du fémur, qui relie la tête au tendon prépubien, la forte lame fibreuse servant d'attache des muscles abdominaux sur le pubis (fig. $\mathbf{n}^{\circ} \mathbf{2}$ ). 


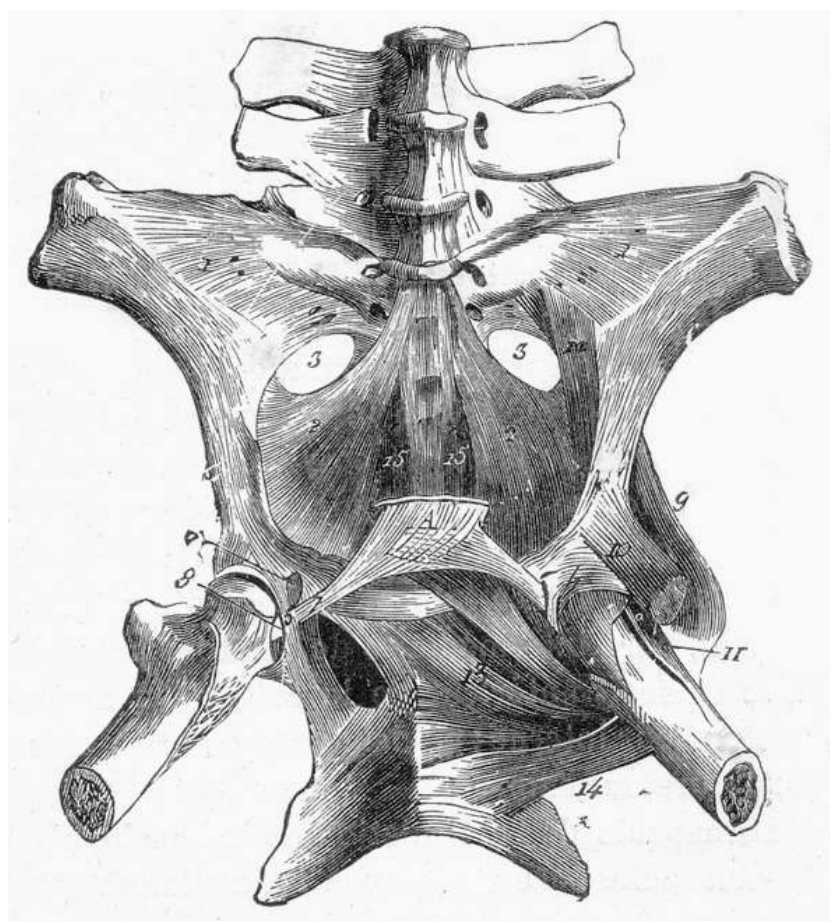

Fig. 170, - Articulations sacro-iliaque et coxo-fémorale, avec les musclés profonds qui enveloppent cette dernière (vue inférieure) *.

Vue de la face ventrale du bassin du cheval montrant le ligament accessoire de la tête du fémur, selon CHAUVEAU, A., ARLOING, S., LESBRE, F.-X. Traité d'anatomie comparée des animaux domestiques. Tome 1. Paris : Baillières, 1905, figure 170.

8 Une modification majeure du squelette concerne le doigt. La main est très allongée, réduite $\mathrm{du}$ point de vue fonctionnel au doigt III, marqué par des phalanges très différenciées, associées en articulations interphalangiennes œuvrant, là encore, principalement dans le plan sagittal. La troisième phalange notamment forme un croissant lié au sabot. L'onguligradie est aussi une condition dont nous avons peine à saisir toute la portée. Il n'y pas de contact direct au sol par une partie sensible comme c'est le cas pour les plantigrades que nous sommes. Et la perception du terrain est obligatoirement plus fruste et plus profonde, assumée par les récepteurs tendineux, ligamentaires ou logés dans le coussinet plantaire (fig. $\mathbf{n}^{\circ} \mathbf{3}$ ) interposé entre le tendon fléchisseur profond et la fourchette. 


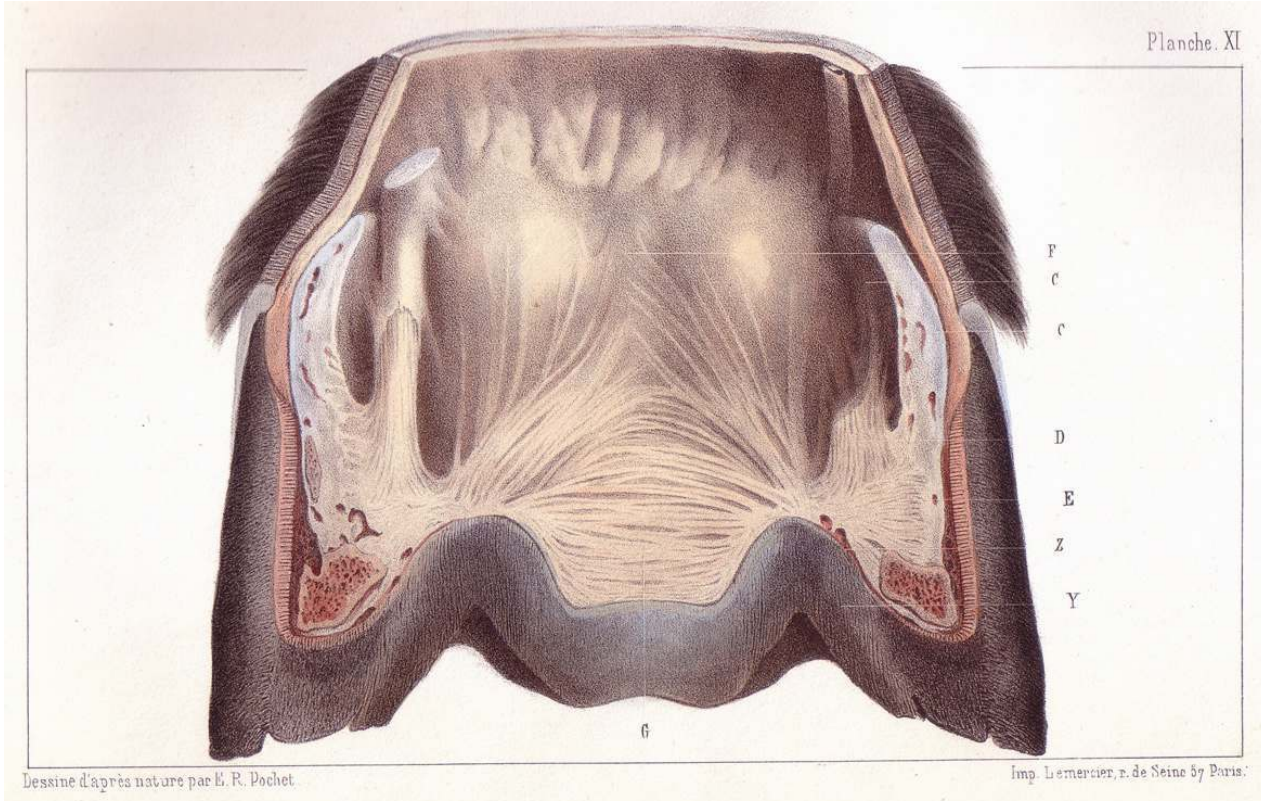

Coupe transversale de la région postérieure du pied, en arrière des phalanges, montrant le coussinet plantaire intercalé entre les cartilages ungulaires, selon BULEY, H. H. Traité de l'organisation du pied du cheval. Atlas. Paris : chez Labé, 1851, planche XI.

\section{Une musculature massive}

9 La modification du squelette s'accompagne de celle de la musculature. Le développement d'une main adaptée à la course se traduit par la concentration des corps charnus dans les régions proximales du membre, et l'absence de ces chefs musculaires dans la main et dans le pied (fig. $\mathbf{n}^{\circ} \mathbf{4}$ ). Cette concentration des masses dans les régions hautes du membre, opposées à des extrémités fines et légères, est un facteur d'économie énergétique. Et la réduction du nombre d'os est associée à une simplification du nombre de muscles, ceux destinés aux doigts autres que le III ou permettant l'abduction/adduction et les rotations externe/interne chez les autres espèces disparaissant. 
Figure 4

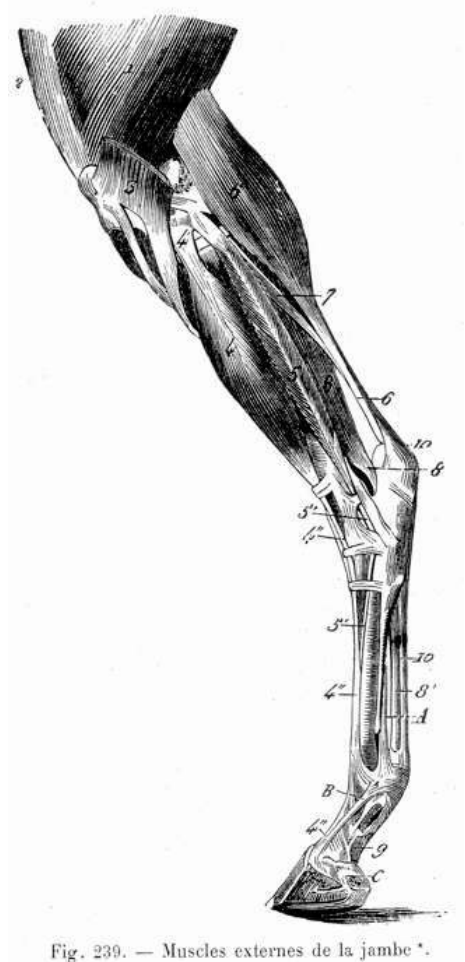

Les muscles du membre pelvien du cheval, selon CHAUVEAU, A., ARLOING, S., LESBRE, F.-X. Traité d'anatomie comparée des animaux domestiques. Tome 1. Paris : Baillières, 1905, figure 239.

Les tendons de ces muscles sont volumineux et pourvus de ligaments accessoires très puissants qui génèrent une automatisation des mouvements et un processus d'amortissement automatisé des appuis au sol (fig. $\mathbf{n}^{\circ} 5$ ). À titre d'exemple, le membre pelvien présente une adaptation tout à fait remarquable et qui témoigne de ce très haut degré de spécialisation: l'appareil réciproque. Celui-ci implique le muscle troisième péronier ou corde fémoro-métatarsienne, situé à l'avant du tibia, et le muscle fléchisseur superficiel des doigts, placé caudalement à ce segment. C'est cet ensemble qui fait que la flexion du grasset s'accompagne de celle du jarret, degré pour degré, et des articulations du boulet et interphalangiennes. C'est aussi ce dispositif qui fait que, lorsque le grasset est bloqué en extension, l'animal peut se reposer sur son membre pelvien. 


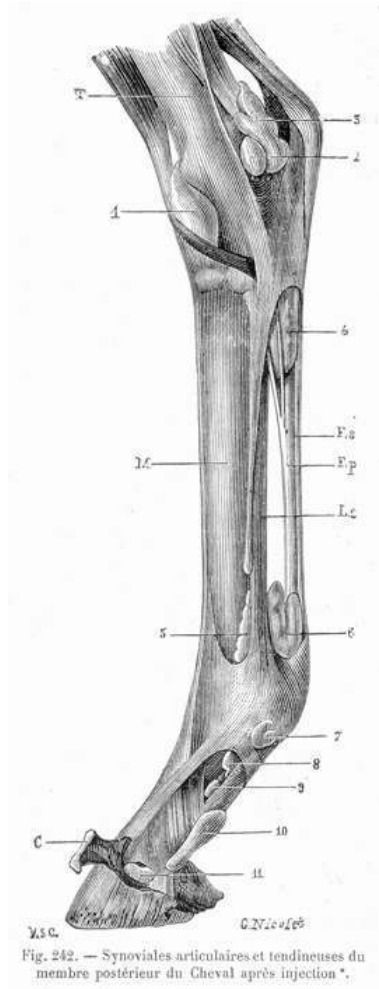

Les muscles de la jambe et du pied du cheval, selon CHAUVEAU, A., ARLOING, S., LESBRE, F.-X. Traité d'anatomie comparée des animaux domestiques. Tome 1. Paris : Baillières, 1905, figure 245.

11 Au bilan, la simplification et la spécialisation des muscles de l'appareil locomoteur conduit à une automatisation des mouvements. Par exemple, la mise en charge du membre dans la première partie de l'appui induit le blocage en extension de l'articulation du carpe tandis que le boulet s'étend et descend du fait de la flexion des deux articulations interphalangiennes. Les tendons et leurs ligaments accessoires s'allongent et, légèrement avant que le canon ne passe la verticale, le mouvement s'inverse tandis que les tendons restituent l'énergie emmagasinée. Et le cycle continue avec le soutien qui conduira à un nouvel appui. La modulation des mouvements doit donc être anticipée et mettre en œuvre les grosses masses situées dans les régions proximales ; une fois engagé, le mouvement implique des flexions et extensions qui ont un caractère inéluctable. Le cavalier et son animal intègrent donc naturellement ces effets.

Un athlète comme le cheval a un besoin considérable en oxygène nécessaire pour alimenter l'activité de ces importantes masses musculaires. Le cœur est très volumineux dans cette espèce, tout comme les artères et les veines qui s'en échappent ou $\mathrm{y}$ parviennent. Les poumons sont aussi très étendus, ont un aspect massif, ne présentent pas une lobation aussi marquée que les autres espèces. L'ensemble forme un formidable système de transport et de régénération gazeuse du sang et est logé dans un thorax très étendu, formé de dix-huit côtes de chaque côté (fig. $\mathbf{n}^{\circ} \mathbf{6}$ ). Une des conséquences de ce thorax si développé est la limitation des capacités de latéroflexion de la colonne vertébrale. Le cheval est un animal assez figé de ce point de vue, et la rigidité de sa colonne thoraco-lombaire nous offre la possibilité de la charger de notre poids. 
Figure 6

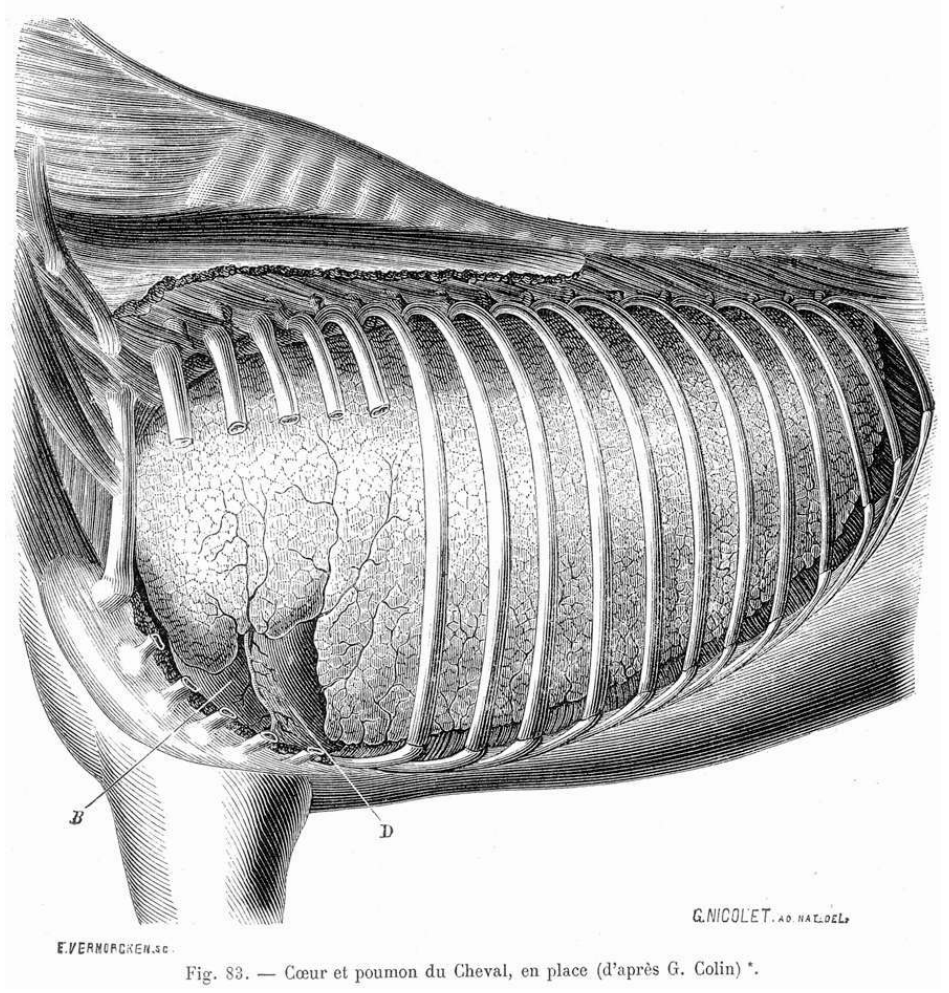

Le cœur et les poumons dans la cage thoracique du cheval, selon CHAUVEAU, A., ARLOING, S., LESBRE, F.-X. Traité d'anatomie comparée des animaux domestiques. Tome 2. Paris : Baillières, 1905, figure 83.

\section{Un système nerveux bien différent de celui de I'homme}

13 Il s'agit là d'un sujet assez peu abordé par les auteurs souhaitant définir les contraintes s'imposant au cheval. Si le système nerveux présente la même organisation globale que chez les autres mammifères, et donc les humains, l'étude de son anatomie fonctionnelle marque les différences adaptatives entre ces deux espèces. On ne peut cependant que regretter que cet animal soit si mal connu sous cet angle-là.

L'encéphale est de taille assez modeste lorsque comparée à celle de l'humain. Le cerveau (télencéphale) pèse en moyenne environ 600 grammes soit $1 / 750^{\mathrm{e}}$ du poids vif (contre 1500 grammes pour l'homme et $1 / 60^{e}$ du poids vif) ${ }^{8}$. L'encéphale dans son ensemble représente $1 / 600^{\mathrm{e}} \mathrm{du}$ poids chez le cheval contre $1 / 50^{\mathrm{e}}$ chez l'homme mais le tronc cérébral reste volumineux. Une différence de taille réside dans les différences de proportions entre le rhinencéphale et le néopallium, chez le cheval où le rhinencéphale est volumineux, et chez nous où il est réduit (fig. $\mathbf{n}^{\circ} 7$ ). 


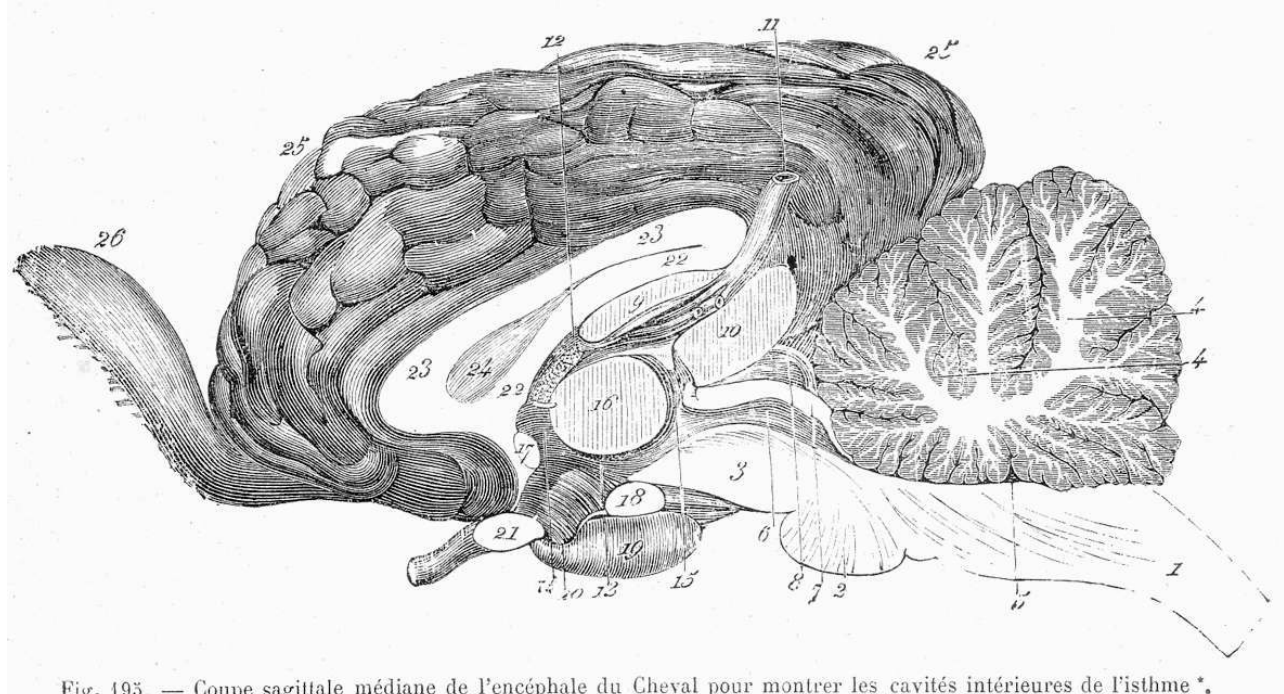

L'encéphale du cheval, coupe sagittale, selon CHAUVEAU, A., ARLOING, S., LESBRE, F.-X. Traité d'anatomie comparée des animaux domestiques. Tome 2. Paris : Baillières, 1905, figure 195.

Autre différence, le cortex sensori-moteur est réduit et notamment le territoire cortical dévolu à la motricité volontaire. Il est particulièrement intéressant d'envisager les différences en matière de gestion de la motricité entre les espèces équine et humaine, à cet égard très différentes. Simplement, on distingue une motricité fine, volontaire, soumise au contrôle de la volonté, et donc d'origine corticale, et une motricité automatique, souvent plus fruste, complétant la première et lui permettant de s'exprimer pleinement. Cette distinction peut être perçue par exemple par la différence entre posture et mouvement ; la première est induite par des tensions musculaires générées par des systèmes peu soumis à la conscience et impliquant la proprioception, et notamment l'équilibre ; la seconde s'appuie sur ce tonus pour développer un mouvement contrôlé par le cortex. L'Humain, avec ses nombreux muscles et ses dix doigts, a fortement développé cette composante du mouvement fin. Dans la moelle épinière, cela paraît au travers du grand développement des tractus cortico-spinaux (fibres allant du cortex aux segments de moelle épinière). La situation est tout autre chez le Cheval, marquée par la réduction extrême des voies de la motricité volontaire au profit de celles dédiées à la motricité automatique. La chose est telle que le tractus cortico-spinal latéral, seul représentant de la voie pyramidale, est formé de fibres de petits calibres se terminant dès le premier segment cervical, aussitôt après leur décussation au niveau des pyramides (dans la moelle allongée). En revanche, le tractus rubro-spinal est bien développé et pourvu de fibres volumineuses; il peut être suivi jusqu'aux segments coccygiens. Et le cordon ventral est occupé par un fort volumineux tractus longitudinal médial, riche de fibres de très gros calibres'. La question du calibre des fibres n'est pas anodine quand on sait que la taille est corrélée à la vitesse de transmission des influx. Ces faisceaux sont issus de formations logées dans le tronc cérébral et fonctionnent sur un mode plus automatique, même si la conscience peut influer sur leur fonctionnement. C'est ainsi que le système nerveux central témoigne aussi de l'automatisation des mouvements, ce qui fait écho à celle observée aux niveaux des systèmes squelettiques et musculaires des membres. 

base du cerveau ou dans le tronc cérébral. Ceci se confirme par la pathologie ; les lésions neurologiques étendues impliquant les hémisphères cérébraux peuvent être bien tolérées dans cette espèce alors que des lésions beaucoup plus faibles conduiraient à des tableaux cliniques dramatiques chez l'Homme. Si des lésions étendues du cerveau sont souvent bien tolérées, celles du tronc cérébral sont souvent gravissimes chez les animaux domestiques.

17 Cette structuration neurologique a des conséquences : le cheval n'est pas doué, comme l'Homme, de la capacité de créer des mouvements conscients fins. Il met essentiellement en œuvre des mouvements automatisés, longuement appris et placés sous le contrôle de centres infra-corticaux. Ceci explique la grande complexité de faire acquérir à un cheval des mouvements inhabituels qu'il soit en outre capable de répéter de façon standardisée.

\section{Que retenir de ces éléments ?}

18 Comment intégrer toutes ces données, leur donner du sens notamment au regard des pratiques d'équitation? La chose n'est pas aisée, d'autant que les données sont rendues parcellaires par le très faible nombre d'études sur les aspects neurologiques, ce qui conduit à l'absence de certitudes. Ce n'est donc pas le lieu de mener une réflexion très détaillée sur les implications sportives de ces données. Cependant, il faut avoir toujours à l'esprit que, comme tout être vivant, le cheval a dû s'adapter pour bénéficier d'une niche écologique garantissant sa survie, et ceci lui a apporté des opportunités dont nous faisons largement usage. Mais ces adaptations se traduisent par des spécialisations limitant d'autres capacités. C'est ainsi que le cheval est très adapté à un mouvement sagittal mais beaucoup moins au déplacement latéral, impliquant de l'abduction/adduction et des rotations externe/interne. Et surtout, tout nouveau mouvement implique un long apprentissage lui permettant de construire un programme moteur qui mettra en jeu les mouvements qu'il peut faire, et seulement ceux-ci.

19 Finalement, les buts de l'art équestre sont bien dictés par ces particularités d'espèce. Les cavaliers, notamment lorsqu'ils sont impliqués dans un processus compétitif, placent leurs compagnons à la limite de leurs capacités et de leurs compétences car, comment le brio des cavaliers pourrait-il s'exprimer dans les choses communes? Comme pour tout phénomène de compétition, le cavalier évolue dans les limites d'un phénomène qui, en l'occurrence, sont celles des capacités du cheval. Il peut exploiter ses aptitudes naturelles à la locomotion rapide et en ligne droite, par exemple dans la course ou la chasse, ou placer sa monture aux limites de ses compétences locomotrices ; c'est typiquement le cas du dressage, et surtout des sauts et des airs relevés, où le cheval doit reproduire, à la demande et de façon standardisée, des mouvements qu'il ne réaliserait pas ou peu à l'état naturel. La difficulté d'acquérir ces programmes moteurs explique la longueur et la difficulté de cet apprentissage.

In fine, connaître le cheval et savoir le replacer dans le contexte de sa niche écologique sont deux impératifs pour qui veut jouir du plaisir de monter à cheval en respectant sa monture et en assurant son bien-être. 


\section{NOTES}

1. L'auteur tient à remercier Bertrand Deputte, professeur d'éthologie à l'École nationale vétérinaire d'Alfort, et Bernard Clerc, professeur d'ophtalmologie dans le même établissement, pour l'aide qu'ils lui ont apportée. Il tient également à remercier Dominique ollivier qui a réalisé un mémoire de stage de master portant sur cette question et a notamment développé deux parties très intéressantes sur les implications de ces adaptations sur les pratiques d'équitation.

2. La majeure partie de ce texte a été publiée dans les actes du $11^{\mathrm{e}}$ colloque de l'École nationale d'Équitation «Posture du cavalier et posture du cheval, leurs particularités et leur influence réciproque" organisé en 2007: DEGUEURCE, Christophe. Le cavalier et son cheval: l'anthropomorphisme est-il possible ? 11ème colloque de l'École Nationale d'Équitation, Saumur, 16 juin 2007. Saumur : École Nationale d'Équitation (ENE), p. 99-105.

3. Décussation : fait que des fibres venant d'un côté traversent le plan médian pour se rendre au côté opposé du corps.

4. PEIFFER, R.L. "Foundations of equine ophtalmology : clinical anatomy and physiology". Equine practice, 1979, 1, p. 46.

5. Fovea: dépression centrale de la rétine dont le fond consiste en une couche de cônes photorécepteurs et au niveau de laquelle l'acuité visuelle est maximale.

6. LEBLANC, M.-A. \& BOUISSOU, M.-F. Cheval qui es-tu? L'éthologie du cheval: du comportement naturel à la vie domestique. Paris : Belin, 2003, p. 56.

7. LEBLANC, M.-A. \& BOUISSOU, M.-F. Cheval qui es-tu? L'éthologie du cheval: du comportement naturel à la vie domestique. Paris : Belin, 2003, p. 57.

8. BARONE, R. \& BORTOLAMI, R. Anatomie comparée des animaux domestiques. Tome $6:$ neurologie 1 (système nerveux central). Paris : Vigot, 2004, p. 398.

9. BARONE, R. \& BORTOLAMI, R. Anatomie comparée des animaux domestiques. Tome $6:$ neurologie 1 (système nerveux central). Paris : Vigot, 2004, p. 159.

\section{RÉSUMÉS}

Les cavaliers utilisent un animal assez éloigné morphologiquement de son ancêtre sauvage mais qui a gardé de son évolution les adaptations qui avaient fait son succès dans sa niche écologique. Sa proximité avec nous a favorisé le développement d'un fort anthropomorphisme vis-à-vis de cette espèce. L'objectif de cette présentation est de montrer quels sont les atouts et les limites dont la Nature a pourvu cet animal, et de montrer comment l'art équestre tire avantage de ces particularités d'espèce.

Riders use horse that are morphologically quite far from their wild ancestors, but who have maintained their evolutionary specificities. Its proximity to us has encouraged the development of a strong anthropomorphism. The objective of this presentation is to show what are the strengths and limitations that the Nature has provided to this animal, and show how the equestrian art takes advantage of these specific adaptations. 
INDEX

Mots-clés : cheval, anatomie, évolution, domestication

\section{AUTEUR}

\section{CHRISTOPHE DEGUEURCE}

Professeur, conservateur du musée Fragonard, Université Paris-Est, École nationale vétérinaire d'Alfort cdegueurce@vet-alfort.fr 